\title{
La micro-imagerie infrarrouge appliquée au diagnostic des cancers cutanés
}

\author{
Elodie LY-MORIN, Olivier PIOT, Michel MANFAIT \\ Équipe MéDIAN « Biophotonique Technologies pour la Santé » \\ UMR CNRS 7369 MEDyC \\ Université de Reims Champagne Ardenne \\ olivier.pioł@univ-reims.fr
}

Parmi les cancers de la peau, les deux types de carcinomes cutanés (basocellulaires et spinocellulaires) sont difficilement distinguables lors de l'examen clinique. Or, il est crucial de les différencier car les seconds nécessitent un traitement et une surveillance spécifiques. Pour réduire au minimum l'intervention humaine, forcément subjective, la micro-imagerie infrarouge présente un potentiel certain lors du diagnostic... à condition de savoir traiter les images des tissus inclus en paraffine qui ont été prélevés. Ce défi a été relevé et est en passe de devenir la prochaine méthode de diagnostic totalement objective et prédictive.

\section{Imagerie spectrale infrarouge}

\section{Principe}

La spectroscopie vibrationnelle d'absorption infrarouge (IR) repose sur l'interaction non destructive entre un rayonnement électromagnétique et la matière. Lorsque le faisceau lumineux qui traverse l'échantillon a une énergie (typiquement de 2,5 à $25 \mu \mathrm{m}$ en longueur d'onde) correspondant à l'énergie entre deux niveaux de vibration de l'échantillon (c'est-à-dire à l'énergie de vibration d'une liaison moléculaire), une absorption des photons de la fréquence correspondante peut avoir lieu.

Un spectre infrarouge représente l'absorbance $(A)$ ou la transmittance $(T)$ en fonction du nombre d'onde $(\sigma)$ qui correspond à l'inverse de la longueur d'onde et est proportionnel à l'énergie du rayonnement, définie par la loi de BouguerBeer Lambert :

$A(\sigma$ ou $\lambda)=-\log (T)=\log \left(\frac{I_{0}}{I}\right)=\varepsilon(\sigma$ ou $\lambda) c d$ où $I_{0}$ et $/$ sont les intensités du rayonnement incident et transmis, $\varepsilon$ est le coefficient d'extinction molaire, $c$ la concentration de la substance absorbante et $d$ l'épaisseur de l'échantillon.

\section{Informations obtenues}

L'absorbance étant proportionnelle à la concentration, il est donc possible

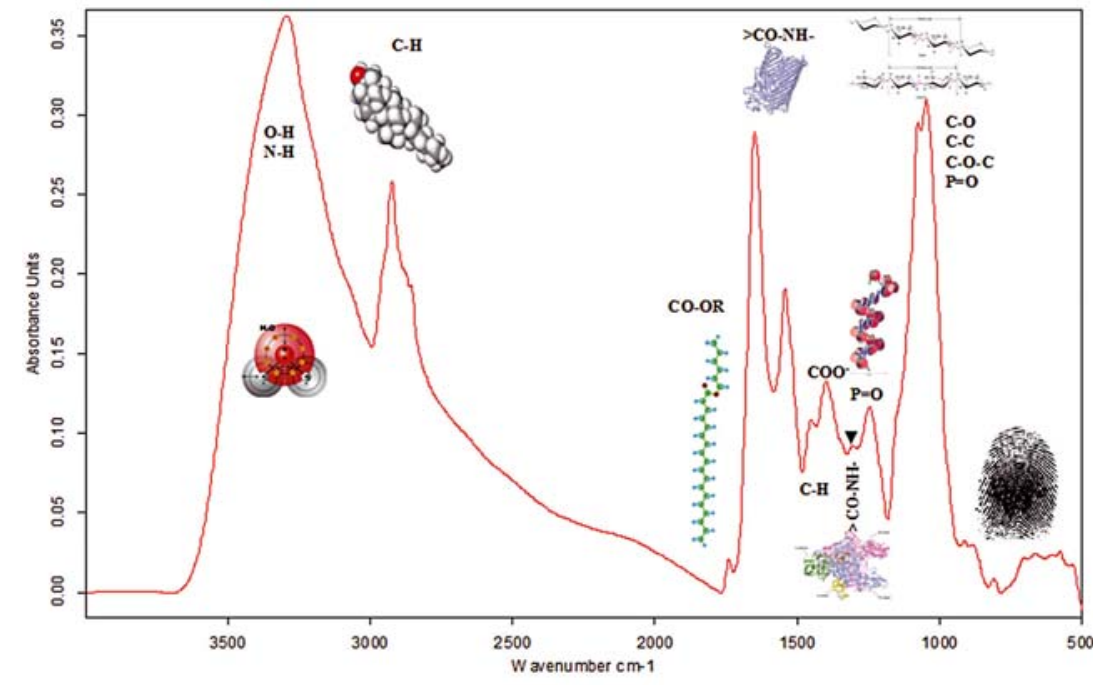

Figure 1. Exemple d'un spectre IR de tissu biologique, à partir duquel des informations concernant les lipides, les protéines et les sucres peuvent être extraites.

d'utiliser la spectroscopie d'absorption IR comme méthode d'analyse quantitative.

Cette technique permet d'obtenir des signaux, très spécifiques, sur la base de la composition biomoléculaire intrinsèque d'un échantillon. Un spectre IR (figure 1) est donc une véritable «empreinte moléculaire» permettant d'identifier et de caractériser un échantillon.

Le couplage d'un spectromètre IR et d'un système d'imagerie permet de réaliser une cartographie chimique d'un échantillon avec une résolution spatiale de l'ordre de 10 microns : les images spectrales peuvent contenir plusieurs dizaines de milliers de spectres, attribuables individuellement à un pixel de l'image spectrale.

Ces images à haut contenu d'information permettent donc d'accéder à des données spatiales et moléculaires/spectrales pour caractériser un tissu complexe, en apportant des informations moléculaires, non accessibles par des analyses histologiques conventionnelles (coloration 
hématoxyline-éosine $\mathrm{HE}$, immuno-marquage), sans marquage ni préparation particulière des coupes tissulaires. Un traitement statistique multivarié des données est donc nécessaire afin de pouvoir mettre en évidence des différences spectrales très subtiles associées à l'état physiopathologique du tissu.

\section{Diagnostic des cancers de la peau}

Parmi les cancers de la peau, on distingue principalement les mélanomes et les carcinomes (les plus fréquents), les carcinomes basocellulaires (BCC) et les carcinomes spinocellulaires (SCC), ont une origine cellulaire commune et sont difficilement distinguables lors de l'examen clinique. Or, il est crucial de les différentier car, contrairement aux BCC, les SCC présentent des risques d'évolution en métastases et nécessitent donc un traitement et une surveillance spécifiques.
Le diagnostic du cancer de la peau s'effectue en deux étapes. Lors du diagnostic clinique, le dermatologue examine la lésion et l'enlève chirurgicalement en cas de suspicion (exérèse). Le prélèvement est ensuite fixé dans le formol, inclus en paraffine, sectionné puis coloré (coloration $\mathrm{HE}$ ) lors du diagnostic histologique. La morphologie du tissu est analysée sous microscope optique par un spécialiste (anatomopathologiste) pour identifier la présence ou non de cancer et déterminer si la lésion a été enlevée dans son intégralité. Le cas échéant, une nouvelle chirurgie doit être programmée, ce qui engendre alors un nouveau coût et un second traumatisme pour le patient.

Ce processus, qui est actuellement la méthode de référence pour la prise en charge des cancers de la peau, repose cependant sur l'expertise des cliniciens et n'est donc pas objectif.

L'objectif de nos travaux est d'évaluer le potentiel de la micro-imagerie infrarouge pour l'aide au diagnostic des cancers de la peau, en particulier pour le diagnostic différentiel BCC/SCC/maladie de Bowen (lésion correspondant à un SCC in situ peu différencié).

\section{Analyse des tissus paraffinés: chimiométrie}

La fixation dans le formol puis l'inclusion dans la paraffine constitue la méthode de conservation des tissus tumoraux la plus utilisée. Ces tissus inclus en paraffine représentent une source considérable d'échantillons ; ils offrent l'opportunité de mener des études rétrospectives permettant de caractériser une pathologie et d'étudier son évolution en suivant le devenir du patient pour identifier des marqueurs spectroscopiques à visée diagnostique et pronostique. Cependant, la paraffine possède un signal IR qui masque en partie le signal du tissu, augmentant ainsi la complexité de l'analyse. Dans ce cas, un déparaffinage chimique à l'aide de

\section{D $5=$ USB 2.0, USB 3.0 ou GigE ? Obtenez tout-en-un !}

\section{Évolution probable de la vision industrielle dans le futur}

Des interfaces distinctes comportent des avantages différents : USB 2.0 est très répandue, USB 3.0 permet des taux de transfert de données élevés et GigE est adaptée aux applications comportant de grandes longueurs de câbles. Encore mieux : IDS Imaging Development Systems, leader sur le marché mondial des caméras USB, a combiné le meilleur de ces interfaces.

A lors qu'USB 3.0 trouve sa place dans la plupart des appareils grand public, qu'elle devienne une norme dans les applications industrielles et l'imagerie professionnelle n'est plus qu'une question de temps. USB 3.0 est l'interface-caméra idéale pour transmettre, à très grande vitesse et en temps réel, des images détaillées sans perte de données. La bande passante disponible est de près de 400 mégaoctets par seconde, soit environ 10 fois celle de USB 2.0 et 4 fois celle de GigE.

Pour garantir la fonctionnalité et la vitesse d'USB 3.0, les caméras professionnelles IDS sont équipées des tout derniers capteurs CMOS, et elles proposent une foule de fonctions supplémentaires. Avec une gamme de boîtiers et des variantes de caméras-carte, à choisir, elles sont parfaites pour toutes sortes d'applications.

Auparavant, les interfaces GigE étaient l'option privilégiée pour « couvrir » les longues distances entre PC et caméra. Désormais, IDS propose des longueurs de câble USB 3.0 pouvant atteindre 50 mètres. « Le câble à fibres optiques est résistant aux interférences électromagnétiques, et un rayon de courbure de $20 \mathrm{~mm}$ est possible », précise Dominik Schillinger, chef de produits chez IDS ; « ce câble actif est compatible avec les caméras USB 2.0, ce qui est rare sur le marché ». Alors pourquoi choisir entre USB 2.0, USB 3.0 ou GigE ? Passez au tout-en-un ! Choisissez USB 3.0 !

IDS Imaging Development Systems Téléphone : +331 42214704 www.ids-imaging.fr 
solvants agressifs est généralement réalisé préalablement à l'analyse spectrale, au risque d'altérer la composition biochimique et la structure des tissus.

\section{Le déparaffinage numérique d'une image spectrale}

Nous avons développé une méthode chimiométrique de traitement de données permettant de déparaffiner «numériquement» les images spectrales IR en adaptant la technique d'EMSC (extended multiplicative signal correction) dont le principe repose sur une minimisation des interférences spectrales (principalement de la paraffine) de façon à ne retenir que la variabilité propre au tissu biologique.

Le point clé de cette correction réside en une modélisation adéquate de la paraffine car son signal n'est pas uniforme à l'échelle d'une coupe tissulaire. Pour modéliser ce signal en tenant compte de sa variabilité, nous avons réalisé une ACP (analyse en composantes principales) sur une coupe de paraffine brute, obtenue selon les conditions standard d'inclusion.

La figure 2 illustre le résultat obtenu à partir d'un échantillon de lésion cutanée paraffinée.

\section{Mise en évidence de zones tissulaires d'intérêt par génération d'images pseudo-couleurs}

Après correction chimiométrique, les données sont traitées par analyse statistique multivariée, par classification selon les k-means. Cette technique présente trois avantages : elle repose sur un algorithme de calcul rapide, ne requiert aucune connaissance préalable sur les données et ne nécessite aucune intervention de l'opérateur dans la classification (il fixe uniquement le nombre de classes ou clusters).

Les spectres sont regroupés en clusters selon un critère de ressemblance basé sur le calcul des distances (généralement de type euclidien) entre les spectres pris deux à deux ; à chaque cluster est ensuite attribuée une couleur. Cette classification permet de reconstruire des images pseudo-couleurs de contraste élevé.
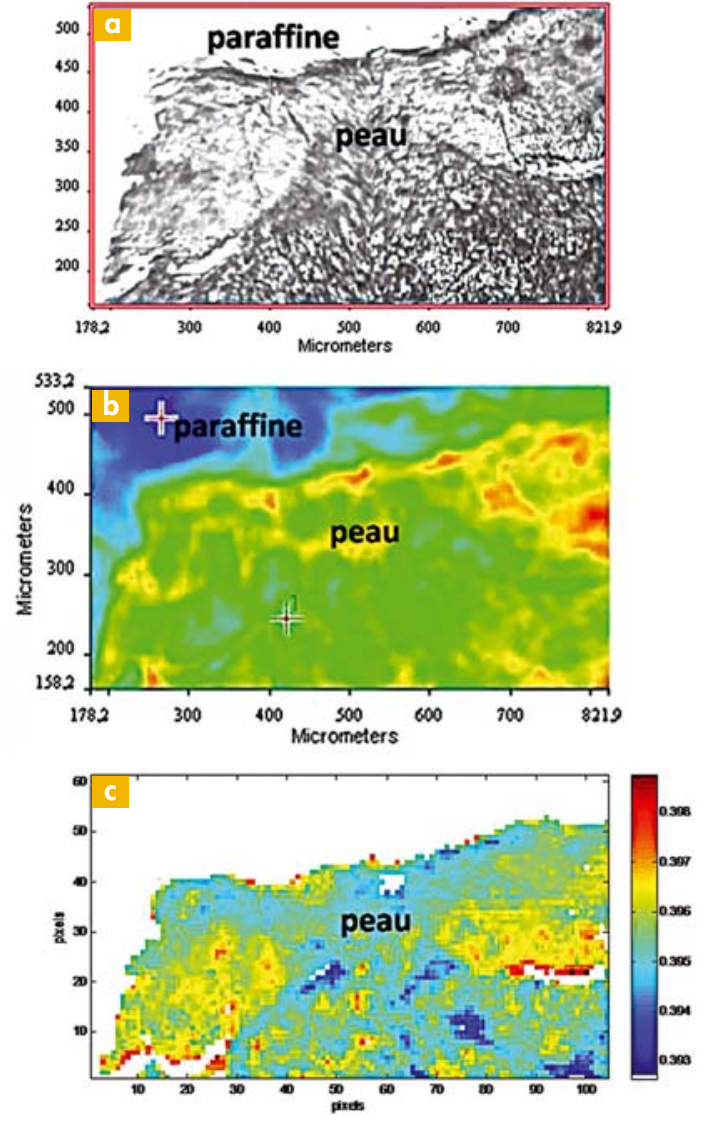

Figure 2. a. Coupe de lésion cutanée paraffinée non colorée $\mathrm{HE}$, incluse en paraffine limage en lumière blanche). $b$. Image spectrale correspondante. c. Image spectrale après déparaffinage numérique. Les pixels en blanc correspondent aux spectres de paraffine seule (sans contribution du tissu) ; ils sont automatiquement retirés par l'algorithme.

Cette analyse, "purement descriptive» dans le sens où elle permet de décrire et de mettre en évidence différentes structures au sein d'un tissu en y associant une signature moléculaire/spectrale, requiert ensuite l'expertise d'un anatomopathologiste pour identifier les structures complexes en comparant les images IR pseudo-couleurs avec les coupes histologiques colorées HE correspondantes. La figure 3 représente un exemple d'images k-means obtenues sur chacun des trois types de lésion (BCC, SCC, maladie de Bowen); chaque échantillon étant traité indépendamment des autres, il n'y a pas de correspondance entre les couleurs d'une image à l'autre. Ces représentations mettent en évidence les différentes structures histologiques des échantillons. Elles permettent non seulement de localiser très précisément les zones tumorales, mais également de révéler une hétérogénéité marquée au sein de la tumeur. De telles informations ne sont pas détectables au niveau des analyses histologiques standard (coloration $\mathrm{HE}$ ), utilisées en routine pour le diagnostic histologique.

De plus, I'analyse de la signature spectrale associée à un cluster permet également d'accéder à des informations sur la composition moléculaire de la structure tissulaire correspondante. Cette démarche permet de construire une banque de marqueurs spectroscopiques spécifiques des différentes structures tissulaires normales, bénignes et/ou malignes présentes au sein des échantillons considérés.

\section{Vers un diagnostic différentiel automatique}

Après l'étape d'attribution cluster/ structure histologique correspondante, le développement d'un modèle de prédiction par LDA (analyse discriminante linéaire) par construction, validation puis test de l'algorithme permet d'analyser en aveugle de nouveaux échantillons dont le diagnostic histologique est inconnu.

L'optimisation des algorithmes a permis d'opter pour un modèle à huit classes, décrivant l'ensemble des structures histologiques présentes au niveau des carcinomes cutanés : épiderme sain, épiderme réactionnel, stratum corneum, stroma, stroma inflammatoire, carcinome basocellulaire, carcinome spinocellulaire, maladie de Bowen.

Un code couleurs simplifié a été adopté: trois couleurs pour les trois types tumeur, une quatrième couleur pour le tissu normal et une dernière pour mettre en évidence les pixels/spectres qui n'ont pas été classés avec certitude (probabilité d'appartenance à la classe inférieure à un seuil de 0,75 ).

La figure 4 représente la classification LDA ainsi obtenue sur un échantillon analysé en aveugle correspondant à un BCC de type nodulaire. En comparaison avec la coupe colorée HE, les zones tumorales sont bien délimitées et correctement identifiées comme BCC (couleur noire). 


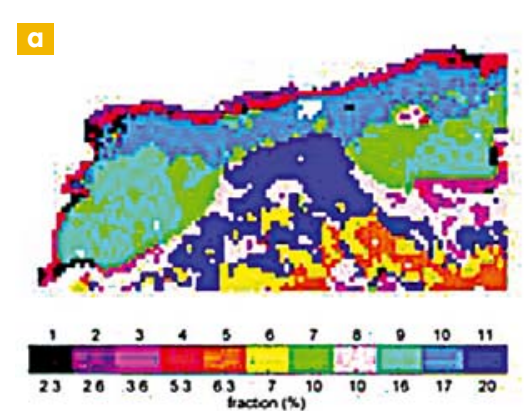

b

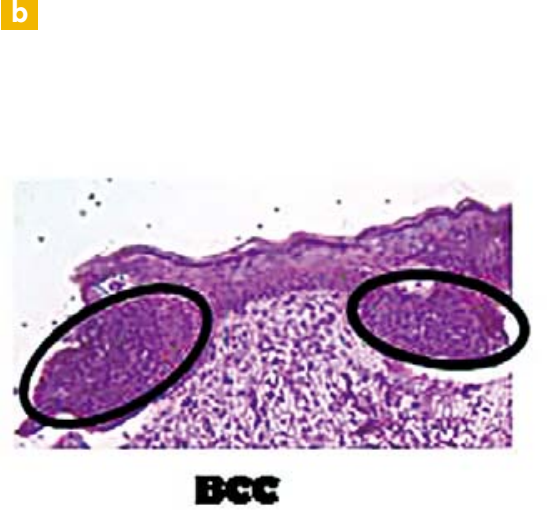

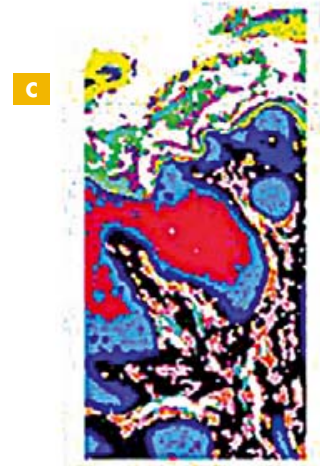
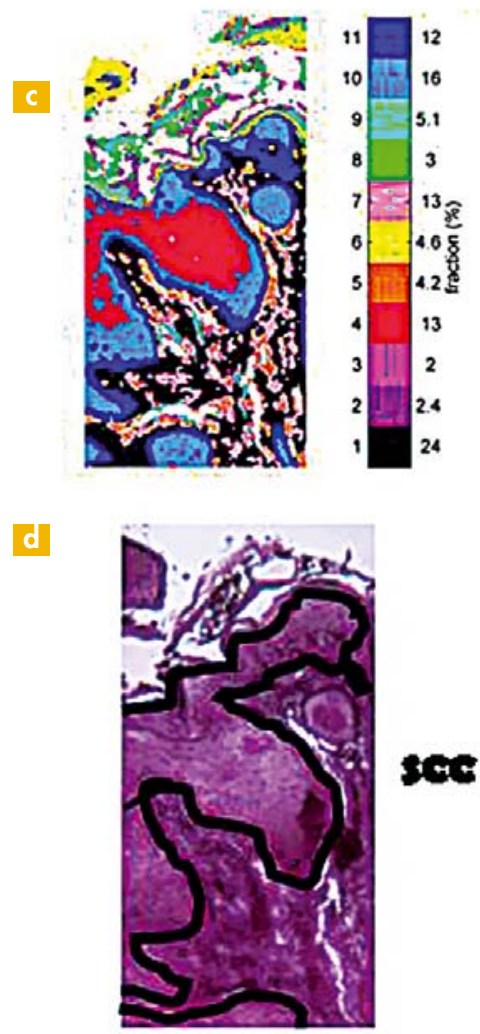

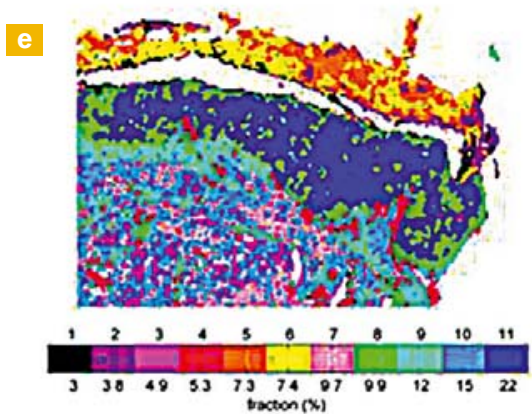

f

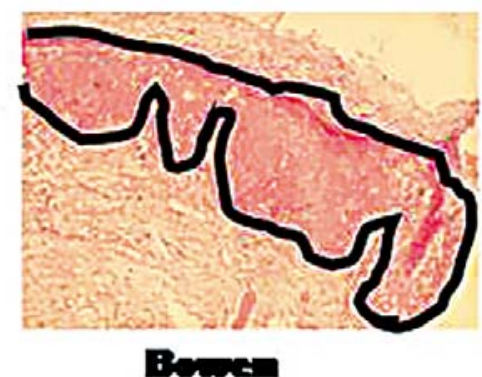

Figure 3. Images pseudo-couleurs obtenues à partir de trois types de lésion ( $a: \mathrm{BCC}, \mathrm{c}: \mathrm{SCC}$ et $e$ : maladie de Bowen) et coupes colorées HE adjacentes correspondantes (b, d et $f$, respectivement). Les zones tumorales sont entourées. Échelle : $200 \mu \mathrm{m}$.

De plus, le modèle indique une bonne spécificité car aucun pixel/spectre de l'image n'est attribué à un SCC ni à la maladie de Bowen. L'histogramme indique que les spectres mal identifiés ( $12 \%$ des pixels de l'image, classe 5) apparaissent au niveau des zones proches de la tumeur, appelée zones péritumorales (couleur bleue), ce qui suggère une composition biomoléculaire particulière au niveau de cette interface.

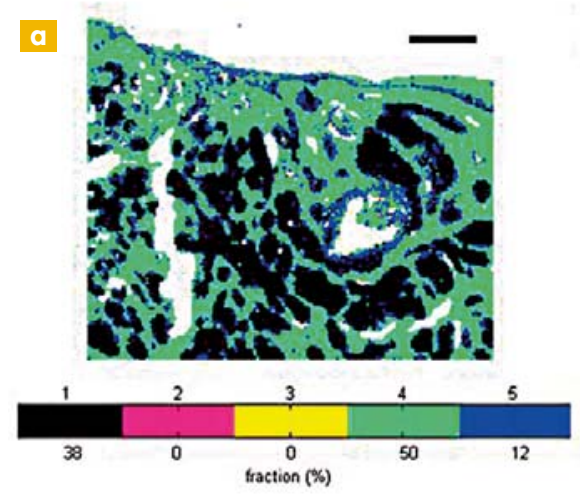

Une fois la banque de marqueurs spectroscopiques constituée, l'analyse par LDA d'une image spectrale d'environ 35000 spectres (soit une zone d'environ $1800 \times 700 \mu \mathrm{m}^{2}$ ) dure moins de dix secondes. Ce processus est automatique et donne un diagnostic totalement objectif. Avec les imageurs infrarouge de nouvelle génération, il est maintenant tout à fait envisageable d'implémenter l'imagerie infrarouge au sein des laboratoires

b

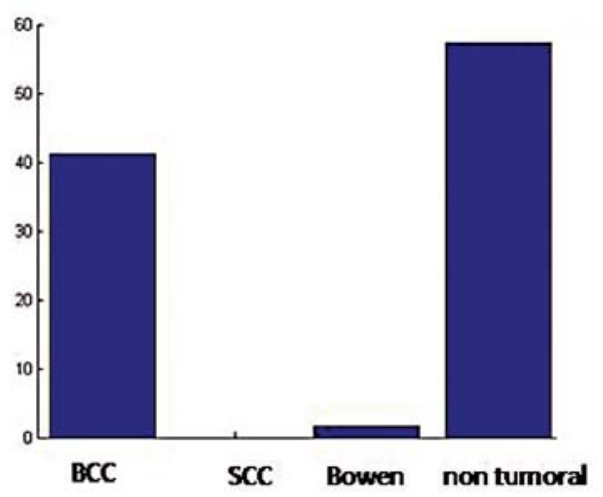

d'anatomopathologie. L'efficacité de cette approche repose sur la pertinence de la banque de données spectrales utilisée pour l'apprentissage du modèle de prédiction. Actuellement, un modèle plus performant est en cours de construction en incluant les annexes cutanées (poils, glandes sébacées...) qui correspondent à des zones de forte prolifération cellulaire. L'algorithme devra distinguer ces annexes de la prolifération tumorale.

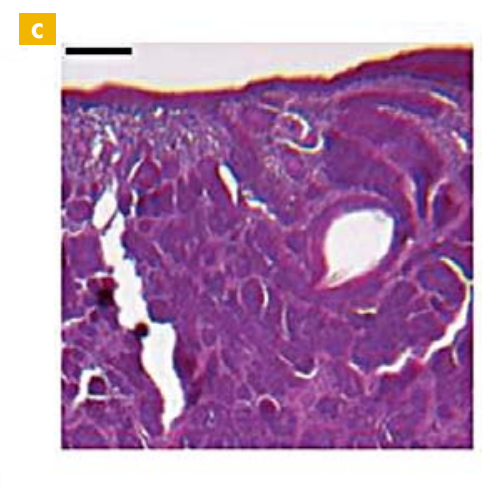

Figure 4. Prédiction LDA. a. Image LDA obtenue sur un échantillon analysé en aveugle (classe 1 : BCC, classe 2 : SCC, classe 3 : maladie de Bowen, classe 4 : non tumoral, classe $5:$ non classé). b. Distribution relative des pixels non identifiés (classe 5) parmi les quatre autres classes LDA. c. Coupe HE correspondante. Échelle : $200 \mu \mathrm{m}$. 